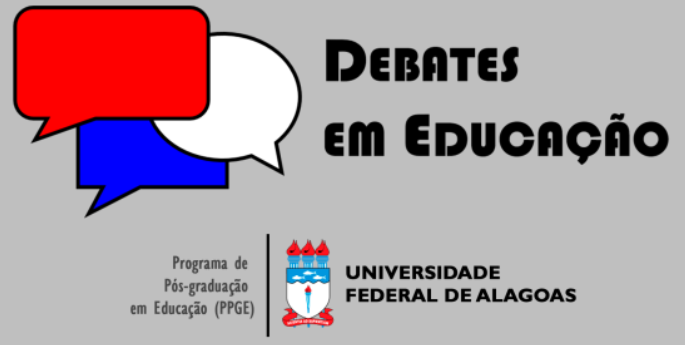

ISSN Eletrônico 2175-6600

Vol. 12 | No. 27 | Maio/Ago. | 2020

Ibrahim Camilo Campos

(9) iD

Université Fédérale d'Alagoas (UFAL) icec.campos@gmail.com

Walter Matias Lima

9 iD

Université Fédérale d'Alagoas (UFAL) waltermatias@gmail.com

\section{RIRE ET STRIDENCE DANS L'ÉDUCATION}

\section{ABSTRAIT}

Le présent article se propose d'exploiter les possibilités et les limites d'incorporation du rire aux processus éducatifs, pratique rare dans les espaces pédagogiques. Pour ce faire, dans un premier temps on s'attachera à rappeler quelques théories sur le rire. Dans un second temps, en s'appuyant essentiellement sur l'infiltration (insertion) et le filtrage (sélection) du rire dans l'ambiance pédagogique, on se penchera sur une critique philo-pédagogique où l'on discute le rapport de transitivité entre la vie et l'école. Tout en prenant compte de la présence du rire dans le discours rhétorique et sa proximité avec la notion de parrhêsia, on défend la problématisation de l'expérience scolaire qui dépasse les limites du logocentrisme et de l'austérité dans l'Éducation.

Mots-clés: Rire. Processus éducatifs. Parrhêsia. Rhétorique.

\section{LAUGHTER AND STRIDENCY IN EDUCATION}

\section{ABSTRACT}

This paper is devoted to explore the possibilities and the limits of incorporating laughter into educational processes, a practice that is scarce in pedagogical spaces. For this purpose, at first, a brief theorization about laughter is made, followed by a philosophical criticism, based on which a transitivity relationship between life and school is defended, in an ethical and aesthetic sense, by infiltration (insertion) and filtering (selection) of laughter in rhetoric for parrhesia, one of the fundamental techniques of self-study studied by Michel Foucault, we argue for a problematization of school experience that goes beyond the limits of the logocentrism and austerity in Education.

Keywords: Laughter. Educational processes Parrhêsia. Rhetoric.

Submetido em: 20/07/2019

Aceito em: 09/03/2020

Publicado em: 22/06/2020

d. http://dx.doi.org/l 0.28998/2 I 75-6600.2020v I 2n27p446-458 


\section{INTRODUCTION}

La dimension épistémologique des processus éducatifs et l'orientation pratique sous-jacente à l'Éducation prennent force dans la mesure où l'on attribue un sens éthique et esthétique à la formation scolaire. D'après une conception philo-pédagogique du rire, rire c'est établir un entrelacement harmonieux avec soi-même. En tant que dérision ou bénévolence, immoralité ou moralité, le rire est provocant, défie la gravité de soi, de l'autre et du monde.

Le but de cet article est de présenter, d'une manière générale, une critique des processus éducatifs logocentriques, tout en examinant la dimension métacognitive de l'enseignement et de l'apprentissage au moyen du rire, pratique sous-estimée et peu présente dans la littérature et dans les espaces éducationnels (LARROSA, 20I3; ARAPIRACA, 2017).

Dans ce travail, cette critique s'articule avec la notion de parrhêsia, outil conceptuel étudié notamment par Michel Foucault (1926-1984), penseur français qui s'appuie essentiellement sur les textes de la philosophie grecque pour prôner les rapports entre vérité morale, franc-parler et pratiques de soi dans l'antiquité gréco-romaine.

Après un survol théorique dans le but de comprendre les rapports de transversalité entre la vie et l'école, dans un sens éthique et esthétique, il nous a semblé utile de tracer quelques grandes lignes sur le rire et sur les pratiques qui en découlent, en envisageant de discuter la polysémie qui le caractérise.

À cette tessiture argumentative initiale succédera une critique de l'absence voire de la quasiabsence de cette dimension vitale - le rire - dans les processus éducatifs. Pour ce qui est de l'intérêt de ce travail, il est suggéré deux approches conceptuelles : celle de l'infiltration (insertion) du rire dans les espace éducatifs et celle de la filtration (sélection) des théories et des pratiques éducatives, surtout en ce qui concerne son application. Notre point de départ théorique est la pratique rhétorique de la parrhêsia.

\section{LE PROPRE DU RIRE, DE PROPRE DE L'HOMME}

George Minois (2003), historien français, auteur de Histoire du rire et de la dérision a déjà souligné la présence timide du rire dans la vie quotidienne.

Nul doute que cette rareté d'un rire libre, cathartique et spontané dans les relations intersubjectives soit le reflet d'une société qui a un faible consensus (MINOIS, 2003), c'est-à-dire, sans un code moral unique, rigide et impératif, car le trait comique est caracterisé par l'opposition avec le sérieux.

En effet, le rire est censé mettre en valeur la différence - le comique, par similitude, opère sous le présupposé de la différence (PROPP, 1992). Dans son ouvrage, Minois (2003) a pris soin de signaler que le rire se glisse sous les fissures de structures axiologiques consolidées. 
Au-delà de la dérision (rire de raillerie), caractérisé par la constante association avec le comique, et qui dévoile les défaillances ou les défauts de qui on rit ou de quoi on rit, le rire peut prendre d'autres aspects assez attirants pour ce travail :

[le rire] peut être joyeux ou triste, bon et indigné, intelligent et bête, vaniteux et cordial, indulgent et sinueux, dépréciatif, timide, aimable et hostile, ironique et sincère, sarcastique et ingénu, tendre et grossier, significatif et gratuit, triomphant, sans pudeur et destructeur. Encore peut-on augmenter cette liste: amusant, mélancolique, nerveux, hystérique, railleur, physiologique, animalesque. Ça peut être un rire ravageur (IURENIEV, 1964 apud PROPP, 1992, p. 28-29).

En dépit de cette diversité, l'affirmation devenue célèbre d'Aristote, selon laquelle « l'être humain est le seul animal qui rit »(ARISTÓTELES, 20I0, p. 135), incrustée par ailleurs dans une explication physiologique - l'échauffement du diaphragme - ne permet pas de conclure, comme le dit Minois (2003), que le rire soit l'essence de l'homme, mais tout simplement que le rire est une potentialité qui lui est inhérente.

Au sein d'une histoire du rire, l'Antiquité a mis en valeur le rire au point d'être considéré comme d'origine divine. Sa presence dans la mythologie aussi bien que dans les satires et les saturnales, par exemple, lui a conféré une valeur positive (MINOIS, 2003)

Le cristianisme, à son tour, a diabolisé le rire en lui donnant un sens négatif, associé au péché originel et à l'éloigment de la perfection particulière de chacun. Le rire, à la limite, était toléré comme une faiblesse humaine, ayant um caractère subversif (MINOIS, 2003).

Au cours de la Renaissance, la théorie classique du rire, chez Aristote, Platon, Cicéron e Quintilien, appuyée notamment sur la dérision, prend un essor considérable. Skinner (2002) examine le développement de cette théorie répandue par les humanistes, lesquels ont associé le rire (en tant que dérision) à la joie et au plaisir. Ils ont aussi mis en valeur l'imprévu et la surprise. Et, en se reportant à la comédie dans la Poétique d'Aristote, ils se bornent à une description des vices risibles, tels que l'avarice et la vanité.

Cependant, quelques écrivains de l'époque s'écartent de la théorie classique et conçoivent le rire hors de sa dimension dérisoire. C'est-à-dire, ils acceptent le rire comme expression de satisfaction et de joie. Cette idée n'a pas été remise en question ; bien au contraire, elle a reçu une adhésion surprenante au début du XVII ${ }^{e}$ siècle. Toutefois, au cours de ce siècle prestigieux, le rire a été banni en raison du discrédit de la psychologie des humeurs et de l'impératif de la bienséance et de l'autocontrôle (SKINNER, 2002).

En 1674, à Rome, l'Académie de la Reine Christine de Suède, il a été proposé au Prêtre Antonio Vieira (1608-1697) et à Girolamo Cattaneo (1620-1685) ) le problème suivant : « si le monde était digne du rire ou de larmes et lequel des deux serait plus sage, c'étaitDémocrite, qui riait trop ; ou Héraclite, qui pleurait trop »(VIEIRA, 17I0, p. 2II) 
Avec sa vision pessimiste du monde, Vieira soutient que Démocrite ne riait jamais, justement parce qu'il riait tout le temps, car le rire naît de la nouveauté ou de l'admiration.

Conformément à la vision de Vieira, Démocrite riait de ce que ne lui plaisait pas. En fait, il pleurait à sa façon, sans larmes, signe de douleur et de tristesse excessives. II riait en pleurant par la bouche, comme s'il était blessé par le monde, il riait avec les pleurs moqueurs du rire même.

En affrontant directement ce problème, Vieira reprend Sénèque et affirme que Démocrite riait à coût sûr des ignorances et qu'Héraclite pleurait des misères. II donne raison à Héraclite, puisque il y a de la misère sans ignorance, tandis que il n'y a pas d'ignorance sans misère.

Un argument est encore exposé par Michel de Montaigne ( I533- I 592). Dans ses Essais, Chapitre L du Livre premier (À propos de Démocrite et Héraclite), Montaigne préfère le rire de Démocrite aux pleurs d'Héraclite. Selon le philosophe français, il y a chez l'homme plus de frivolité que malheur, plus de sottise que méchanceté (Montaigne, 2009).

La polysémie du rire se définit et se fait comprendre par le truchement de l'intentionnalité subjective de l'individu qui le déclenche et qui se lance contre autrui. Le rire touche alors la raillerie sardonique. En faisant usage de la dérision, le rire prend alors des distances afin de vaincre l'autrui, affirme Thomas Hobbes (I558-1679). Par contre, dans sa poursuite vers et envers l'autre, avec la finalité de s'approcher de lui et le vaincre en s'amusant, il se revêt de légèreté spirituelle. Cet amusement demande de l'altérité.

À ces deux positions diamétralement opposées, voire inconciliables, correspondent, respectivement, ce que Pavis nomme « le rire d'accueil » et « le rire d'exclusion » (PAVIS, 1999, p. 59-60). Dans l'article 178, des Passions de l'âme, René Descartes (I596-1650) étudie et approfondit «le rire d'exclusion » et en déduit que la raillerie est une sorte de joie mélangée avec de la haine :

La dérision ou moquerie est une espèce de joie mêlée de haine, qui vient de ce qu'on aperçoit quelque petit mal en une personne qu'on pense en être digne : on a de la haine pour ce mal, on a de la joie de le voir en celui qui en est digne. Et lorsque cela survient inopinément, la surprise de l'admiration est cause qu'on s'éclate de rire (...) (DESCARTES, 2015, p. 22I).

Ainsi, le rire peut agréger ou désagréger, construire ou détruire, catalyser ou atténuer la violence. Faiblesse édifiante ou destructrice, éticité ou raillerie, une prosopopée du rire s'attacherait plutôt à des ambiances lotiques, à des températures glacées ou torrides, tout aux prises à l'éphémérité et au volatile.

Dans son oeuvre Le rire: essai sur la signification du comique, Bergson (1991) privilégie le rire moqueur (railleur), social et qui réprime, un rire plein de froideur par rapport à l'autrui, qui se réalise grâce à l'intelligence, vocable dont l'étymologie, nous assure Onfray (2002), signifie la capacité de mettre en relation ce qui à première vue semblait séparé, et qui appelle le curieux, l'amusant, l'inattendu et le singulier.

Selon ce philosophe français, cet aspect du rire fonctionne comme " une brimade sociale » (BERGSON, 1991, p. 103). Injuste, malveillant et « fait pour humilier, il doit donner à la personne qui en est l'objet une impression pénible. La société se venge par lui des libertés qu'on a prises avec elle» 
(BERGSON, 1991, p. 150). Bien entendu, il ne saurait être question que ce n'est pas de cette sorte de ce rire que l'Éducation en a besoin.

Néanmoins, en tant que pratique sociale, se plaçant entre deux extrémités, «l'amour inconditionnel et la lutte à mort » (POIZAT, 2002, p. 5), le rire, sans utopies et sans violence poussées à l'extrême, trace la voie vers la coexistence avec l'autre en société (PERELMAN; OLBRECHT-TYTECA, 2005).

Cette polysémie du rire entraîne une pluralité de valeurs présents dans les rapports à soi-même et aux autres. En ce sens, la stridence du rire peut déranger l'ordre scolaire mais aussi réveiller les sujets éducatifs en vue de valoriser les expériences émotionelles comme composante fondamentale d'une Éducation attentive et soigneuse des rapports de transitivité entre la vie et l'école. II faut qu'il y ait, donc, une infiltration et un filtrage du rire dans les théories et dans les espaces éducationnels.

\section{POUR UNE INFILTRATION ET UN FILTRAGE DU RIRE DANS LES THEORIES ET DANS LES ESPACES EDUCATIONNELS}

Larrosa (2013) estime que la faible utilisation du comique dans le champ de la pédagogie se doit à un moralisme excessif, qui mène assurément au sérieux et à l'austérité, ainsi qu'à un ferme optimisme pédagogique. Selon l'auteur espagnol (2013, p. 168), « un professeur a quelque chose d'un prêcheur. Pour cela, le ton professoral est un mélange d'austérité et de dogmatisme », voire, peut-être surtout, au plan moral.

Le rire qu'exalte Larrosa n'est pas le rire neutre du loisir, le rire protecteur de remparts ni le rire qui sépare, mais celui qui, en tant que « composante dialogique du sérieux » (LARROSA, 20 I3, p. 170), prend une double fonction, orientée vers une problématisation des enjeux éducatifs au plan éthique.

La première consiste à relativiser et à défaire - sous l'extériorité délibérément déguisée (le langage du comme si) - les discours convenus qui arborent les masques que chacun porte dans la vie en société.

La deuxième fonction du rire dans le champ pédagogique consisterait à promouvoir l'auto-ironie, permettant de cette sorte une distance ou une décentralisation du sujet par rapport à lui-même. II est possible d'inclure l'humeur dans cette dimension, dû à la proximité entre ces deux catégories, l'humeur et le rire.

II nous importe maintenant de brosser un tableau théorique plus ample, dans lequel le rire puisse intégrer l'enseignement et l'apprentissage, c'est-à-dire, un rire qui dépasse les aspects cognitifs et favorise des sphères relationnelles plus authentiques et institutionnellement moins délimitées.

Le rire, sous cette optique, peut être compris dans les processus éducatifs comme un élément de double transitivité entre la vie et l'école (PAGNI, 20I I). 
La première direction part de la vie à l'école par l'insertion du rire, en tant que pratique socioculturelle, à l'intérieur des lacunes des cadres scolaires, constitués par des verticalités hétéronomiques qui suffoquent la subjectivité de l'apprenant et de l'éducateur. Cela se fait sous un ethos pédagogique de caractère instrumental et homogénéisé en ce qui concerne les aspects de la subjectivité, du temps et de l'espace.

Mutatis mutandis, à ce sujet nous n'avons pas mieux à faire qu'à rappeler Quintilien (35-100 apr. J.C.), rhéteur romain qui, trouvant ses fondements dans la rhétorique, prend la défense de l'utilisation sociale du rire. Quintilien met en évidence le fait suivant :

qu'il n'y a pour cela ni exercice ni enseignement. II est vrai qu'à table et dans la conversation on rencontre beaucoup de gens qui savent lancer des plaisanteries ; mais cet art s'apprend dans le commerce du monde, tandis que la raillerie oratoire est rarement d'usage, qu'elle n'est pas l'objet d'un enseignement particulier, et qu'on renvoie à l'école du monde ceux qui veulent s'y exercer (QUINTILIEN, I842, p. 224).

Vient ensuite un processus de filtrage (contrôle) pédagogique-curriculaire, aux dimensions éthique et esthétique, dans lesquelles le rire dérisoire, négatif et grégaire est retenu et séparé des rires caractérisés par la sensibilité envers l'autre et par une autocritique modeste au sein des pratiques éducatives En ce sens, il apparaît à l'évidence, comme le dit Bergson ( 199|), que la vanité est l'élément fondamental de la dérision.

Le deuxième sens de la transitivité part de l'école et va vers la vie, de façon que, selon les critères déjà définis, l'organisation de l'expérience éducative soit projetée et réalisée dans les existences concrètes et quotidiennes des sujets apprenants, confrontés à la haine et à la violence, visibles ou occultées dans la vie quotidienne brésilienne.

II ne s'agit pas de faire de l'école une arène de cirque, ni de la considérer uniquement comme un lieu de loisir. Comme le dit Larrosa (20/3), il s'agit, en outre, d'incorporer la dimension du rire aux processus éducatifs, de le concevoir comme un désordre bienvenu qui favorise l'émergence de subjectivités et de créativités. Le rire est expérience, le rire nous oblige à penser, affirme fortement Larrosa. Cela n'implique pas la dévalorisation de la réflexion critique.

En ce sens, l'étrangeté et la clairvoyance propres au rire (ARAPIRACA, 2017) - ces éléments qui dépassent le champ de l'acquisition de compétences cognitives - peuvent être en quelque sorte exploitées et vécues afin de problématiser l'expérience dans les ambiances éducatives.

Le rire est la constatation de tout ce qui est hors du commun, incroyable, extravagant, différent ou surprenant. Toutefois les ambiances scolaires - fortement régies par des normes réglant le temps, l'espace, autant que les contenus et les pratiques scolaires - sortent largement du ce cadre. Les événements ne se transforment pas en expériences, bien au contraire, ils deviennent tout simplement des moyens scolaires formés, eux, par une culture scientifique, logocentrique, formelle et objective laquelle tourne le dos aux 
joies et aux douleurs réellement vécues (d'où le caractère insulaire de l'école). Tout cela, à la limite, gère, de la part des étudiants, un manque d'intérêt et une absence de liaison affective vis-à-vis de l'école.

C'est en ce sens que nous pouvons rappeler Quintilien (De Risu, § 19). De même que le sel donne de la saveur aux plats, dit-il, en méprisant le dicton trop de rire, peu de sagesse (muito riso, pouco siso, en portugais), le rire suscite la soif chez qui l'écoute.

Fondé sur une étude ethnographique, menée dans un centre scolaire de la banlieue de Barcelone, en Espagne, et fréquenté par des adolescents ayant des problèmes scolaires, Martín (2006) a observé l'utilisation que les éducateurs font aussi du rire que de l'humeur en tant que stratégies pédagogiques.

On peut ramener la thèse de Martin à quelques modalités pédagogiques du rire : I) une réponse à des provocations des élèves en vue d'atténuer les situations et les comportements négatifs ; 2) un défi pour la réalisation de pratiques éducatives et pour l'acquisition d'interlocution sociale; 3) désapprobation et réorientation de comportements adéquats sans qu'il y ait des dégâts issus des conflits; et 4) démonstrations d'affectivité et de proximité relationnelle. « En riant, on dit des choses sérieuses » (ridendo dicere severum), affirme Nietzsche dans son oeuvre autobiographique.

Le rire tend, donc, à déplacer l'attention et la tension situationnelle vers un autre état de spiritualité, et cela permet l'inhibition de la violence dans les ambiances scolaires. En somme, il est un élément potentiel pour résoudre les conflits. Cela atteint directement les personnes concernées. En d'autres mots, tout se résout sans la participation neutre d'un tiers (MARTíN, 2006).

Outre cette technique, il faut penser à une dimension plus vaste du rire et du sens commun de l'humeur. Le rire ne fonctionne pas comme atténuation du conflit (MARTíN, 2006), mais comme une attitude existentielle enracinée dans l'expérience du visible : la sensibilité de l'éloignement implique, pour sa part, un éloignement de la sensibilité. L'utilisation de ces deux catégories - le rire et l'humeur - peut avoir une efficacité plus grande qu'une pitié hypocrite qui recherche un point d'appui dans l'autrui lequel se veut être reconnu et se faire reconnu comme un vertueux.

Cette discussion mérite une réflexion. Le problème qui se pose alors est d'essayer d'examiner la synergie entre le rire et l'éticité dans le domaine des espaces scolaires et de savoir quels postulats théoriques et pratiques peuvent être canalisés pour développer le potentiel critique sur soi-même et sur le monde. En un mot, il faut être attentif à cette réalité de l'expérience humaine qu'est le rire. 


\section{DU RIRE A LA STRIDENCE DE LA PARRHESIA DANS L'ÉDUCATION}

\section{I Parrhêsia, vérité et discursivité}

Les enjeux éducatifs à l'égard de l'expérience du rire sous une dimension éthique peuvent être enrichis lorsqu'on y rajoute des techniques du souci de soi, notamment, celle de la parrhêsia. Le thème de la parrhêsia a été abordé par Foucault dans de divers travaux et dans des cours donnés en France et ailleurs. II s'agit du vocable grec ( $\pi \alpha \rho \rho n \sigma i \alpha)$, établi en latin parrhesia. Cette notion de parrhêsia, au cours des siècles, depuis l'antiquité gréco-romaine jusqu'à l'époque médiévale, a pris de nombreux sens dans plusieurs domaines (politique, philosophique et religieux). Dans l'antiquité, la parrhêsia n'était pas considérée une catégorie de réflexion autonome, excepté le Traité de Philodème (Peri Parrhêsias) (FOUCAULT, 2010).

Dans une conférence proférée à l'Université de Berkeley, en 1983, Foucault ainsi la définit :

(...) parrhesia est une activité verbale dans laquelle l'orateur exprime son rapport personnel à la vérité, et il risque sa vie parce qu'il reconnaît le dire-vrai comme un devoir pour améliorer ou aider les autres (ainsi que pour soi-même). Dans la parrhesia, l'orateur fait usage de sa liberté et choisit le franc-parler au lieu de la persuasion, la vérité au lieu de fausseté ou du silence, le risque de la mort au lieu de la vie et de la sécurité, la critique au lieu de l'adulation, et le devoir morale au lieu de son propre intérêt et de l'apathie morale (FOUCAULT, 200 I, p. 19-20. Traduction libre).

La vérité de la parrhêsia garde un sens plutôt moral, loin du discours raisonneur. Le dire vrai et franc constitue une forme de vie de celui qui le pratique, puisque le logos se fond dans l'existence même du sujet (FOUCAULT, 20I2). En ce sens, la parrhêsia est métadiscursive, puisqu'elle est un comportement du sujet qui philosophe; elle est présente dans sa manière de s'habiller et dans son mode d'être (FOUCAULT, 2008).

Apparemment centrée sur la figure du maître, la parrhêsia est un jeu entre deux personnages : elle se développe entre le maître et son disciple, celui-ci ayant la tâche de provoquer la voix du maitre et celuilà de répondre à sa provocation. Si d'un côté, rappelons-le, il y a une parrhêsia qui impose le silence au disciple, de l'autre côté il y en a une qui prêche l'ouverture réciproque (FOUCAULT, 20 I2), notamment chez Sénèque, qui conçoit une « sorte d'obligation double où les deux âmes échangent, par rapport à la vérité qui se dit, leur propre expérience, leurs propres imperfections, et où elles s'ouvrent l'une à l'autre » (FOUCALT, 2012, p. 183).

Le rire pourrait-il être utilisé dans la pratique philosophique de la parrhêsia ? Au cas affirmatif, il nous faut poursuivre l'investigation : de quel rire s'agit-il ?

Un avant-propos dès lors s'impose. II ne s'agit pas de fureter dans le passé une catégorie conceptuelle et pratique comme une panacée propre aux discours éducationnels de salut, mais plutôt de 
réhabiliter cette catégorie sans avoir pour autant prétendre un retour à son identité. Loin d'un transfert artificiel, ce qu'on prétend c'est l'utiliser en tant qu'un outil théorique indispensable pour la construction de pratiques éducatives.

L'un des courants philosophiques de l'antiquité qui fait de la dérision une composante extrêmement importante dans la réalisation d'une vie savante et authentique - une vie animale (canine) car débarrassée des règles sociales - c'est le cynisme. Diogène de Sinope (V-IV av. J.C.) est l'auteur de curieux récits contenus dans l'ouvrage Vies et doctrines des philosophes illustres, de l'homonyme Diogène Laërce, historien et biographe grec, daté du tournant des II-III siècles de notre ère.

Les rapports entre dérision et parrhêsia, qui, dans cette école philosophique passent ouvertement par l'insulte, par la moquerie et par la pratique publique d'une vie menée en conformité avec la nature, ne se trouvent pas dans les traditions platonicienne et aristotélicienne ni dans la tradition philosophique chrétienne moyenâgeuse qui a rechassé le rire (MINOIS, 2003).

II ne faut d'ailleurs pas manquer d'ajouter que la parrhêsia cynique incorpore le rire ravageur, grossier, frontal et agressif. Notre investigation ne prend pas cette voie. Elle cherche plutôt à faire usage de la rhétorique du rire en tant qu'une composante auxiliaire de la pratique de la parrhêsia.

\subsection{Du rire rhétorique au rire de la parrhêsia}

L'indissociabilité entre le logos et le bios du parrésiaste, dont on a parlé plus haut, constitue l'une des principales différences entre la parrhêsia et la rhétorique. La rhétorique a pour visée persuader au moyen du discours. Fonctionnelle, elle n'a pas de profondeur dans sa structure (REBOUL, 2004). Sa surface, toutefois, comporte une unité entre forme et fond (REBOUL, 2004)

La rhétorique, écrit Glansdorff (2010, p. 75), est une « technique de surface dont l'apparence de vérité ne suffit pas à le fonder comme discours vrai ». Elle est quand même utile au discours parrésiaste, par ses compétences acquises et placées dans l'ethos (dimension centré sur l'orateur) ou dans le logos (dimension centrée sur la rationalité du discours).

En tant que technique argumentative puissante, le rire, parmi d'autres utilisations, sert à refuser l'adhésion à un présupposé du discours. Ou de même à refuser l'adhésion à deux thèses incompatibles (PERELMAN; OLBRECHTS-TYTECA, 2005).

Tout en affirmant les différences entre parrhêsia et rhétorique, Foucault (2010) fait ressortir ce qu'il y a d'irréductible entre eux, au sens où la première se présente surtout comme le vrai-dire, tandis que la deuxième est un art ou une technique visant à persuader, sans que la vérité soit un élément essentiel du discours. 
Dans cet article, on n'étudie pas la parrhêsia comme figure rhétorique (oratio libera), ni comme dépouillement ou liberté de formes (le degré zéro de la rhétorique). Nous prenons le chemin l'inverse. Ici, la rhétorique du rire est employée comme élément d'appui du discours parrésiaste, aidée par l'apport théorique présenté par Cicéron ( I06-43 a.v. J.C.) et par Quintilien (35-100 apr. J.C.).

Nous sommes d'avis que les espèces de genre ridicule, dans l'art oratoire, peuvent être utilisées de façon compatible avec la bienveillance parrésiaste. Bien que la parrhêsia conduise le disciple à la vérité de soi-même, elle n'a pas pour objectif le convaincre.

Deux ouvrages rhétoriques sur le rire, écrits pendant l'antiquité romaine, sont considérées, parmi d'autres, comme les premiers textes qui systématisent le rire dans la pensée occidentale (ALBERTI, 2002). II ne faut pas oublier les textes disparus, à l'exemple du Livre II de la Poétique d'Aristote.

Le rire, dans l'oeuvre de Cicéron, est présent dans De l'Orateur (De l'oratore), du Livre II (De ridiculis), alinéas 216 à 291, dans lequel le personnage César cherche à répondre à cinq questions : qu'estce que le rire? Où se trouve-t-il ? L'orateur doit-il provoquer le rire ? Dans quelle mesure doit-on l'utiliser ? Quels sont les genres qui en découlent ? (§ 235).

Dans l'oeuvre de Quintilien, le rire est étudié dans l'Institution Oratoire (Institutio oratoria), du Livre VI, chapitre III (De risu). Quintilien a reçu de fortes influences de l'oeuvre cicéronienne.

Pour Cicéron, le rire s'origine de la turpitude ou de la déformation (§ 236), des vices (§§ 238 et 239), voire d'une quasi-laideur, sous l'aspect moral aussi (§ 248). Quintilien y ajoute une composante : l'imperfection. En ce sens, selon Quintilien, le rire est, d'une manière générale, faux, tordu et inférieur (§ 6).

Il est proche d'un rire sournois, méchant, bien qu'on puisse rire avec élégance comme il arrive dans l'art poétique.

En plus, le rire peut être lascif, injurieux, moqueur, grossier ou subtil (§ 27). II ne s'oppose pas à l'urbanité. D'après Quintilien (1977, p. 62) :

(...) certains mots, même plaisants, peuvent figurer parmi ceux qui ne sont pas suffisamment de bon ton. Au vrai, à mon sens, le bon ton suppose qu'il n'y ait rien qui détonne, rien de campagnard, rien de fade, rien d'étranger ni dans la pensée, ni dans les mots, ni dans la voix ou le geste, si bien qu'il réside moins dans les mots isolés que dans la tonalité générale de l'énoncé (...).

En vantant le rire et son utilisation dans le discours oratoire, Cicéron, dans l'alinéa 236 du De ridiculis, conclut que le rire et indispensable à l'orateur:

(...) la gaité dispose à la bienveillance en faveur de celui que l'inspire ; un trait d'esprit, un bon mot surprend agréablement l'auditeur et lui fait admirer celui qui le trouve, surtout lorsque c'est pour se défendre, quelquefois même lorsqu'il attaque ; la bonne plaisanterie déconcerte un adversaire, l'embarrasse, l'affaiblit, l'intimide, le réfute ; elle fait regarder l'orateur comme un homme bien élevé, instruit, de bon goût ; elle a surtout le grand avantage d'adoucir, de relâcher la tristesse et la sévérité, de tourner en plaisanterie, de faire oublier par une saillie des imputations graves et odieuses qu'il serait souvent difficile de repousser par le raisonnement (CICÉRON, I830, p. 43I). 
Selon la pensée de Quintilien, inscrite dans cet ouvrage, le rire écarte les sentiments tristes, détourne l'attention des auditeurs vis-à-vis des faits, encourage le public, fatigué d'accompagner le processus argumentatif du discours $(\S ।$ ). Le rire sert aussi à amortir les situations graves, entourées de haine.

Quant aux limites de l'usage du rire dans l'art oratoire, Cicéron conseille la modération afin d'éviter la méchanceté et attire l'attention pour le respect d'autrui (§ 237). On doit surtout éviter l'usage fréquent d'imitations (§ 25।), de grimaces ou d'obscénités dans des endroits inadéquats.

Ainsi, Quintilien conseille de ne pas employer le rire d'une façon explicite ou exagérée (§§ 26 et 30), dans des endroits qui ne peuvent pas convenir à sa pratique, et, non plus, d'une manière qui puisse blesser les personnes (§§ 29 et 33). En outre, Quintilien dit qu'il faut rejeter le rire prémédité, arrogant, pétulant (§ 33).

En ce qui concerne les genres du ridicule, Cicéron et Quintilien présentent un répertoire multiple et varié : blague, métaphore, ironie, allégorie, ambigüité, hyperbole, répréhension des dires, simulation, malice, interposition de vers, utilisation de proverbes ou de phrases insolites, chute de l'expectative, jeux de mots, utilisation d'ambigüités et de similitudes.

II s'agit de genres qui peuvent être mobilisés ludiquement en vue de lier le franc-parler de la parrhêsia avec l'expérience éthique et esthétique du rire,démuni de dérision, tout en surmontant la neutralité technique qui constitue le discours rhétorique. En ce sens, le rire se deploie comme une des formes éducatives de la parrhêsia, en tant que pratique métadiscursive.

Donc, il se peut que, dans le domaine de l'Éducation, l'apport de la rhétorique, enrichie d'un telos parrésiaste, dans le domaine de l'Éducation scolaire, élève la qualité des rapports interdisciplinaires, en fonctionnant comme un élément de transitivité entre la vie et l'école. Cela permettra la construction de vérités et d'affectivités.

\section{CONCLUSION}

En effaçant ce qui en principe n'est pas facile à diluer en arguments, le rire favorise chez les sujets un état d'esprit qui surpasse la rationalité des pratiques et des théories éducatives. En effet, l'épicentre du rire n'est pas du tout dans la théorisation ni dans scientificité des processus éducatifs (CITOT, 2002), mais dans l'explosion de vitalité et de spontanéité, sans une rationalité éducationnelle dogmatique, austère et contraire à une praxis de transitivité entre la vie et l'école.

L'infiltration (insertion) et le filtrage (contrôle) du rire dans les théories, les pratiques et les espaces éducatifs permettent de diminuer les tensions et les disputes. Nourries de sérieux, ces pratiques rabaissent 
le comique à condition de superficialité. L'infiltration et le contrôle contribuent également à l'exercice de l'auto-ironie et à la relativisation de l'austérité avec soi-même et avec l'autre.

La rhétorique garde une relation étroite avec cette pluralité. Elle met en évidence la nécessité de comprendre les valeurs de l'autre. Appuyées sur la notion de parrhêsia, les recherches de cet ordre doivent privilégier non seulement les enfants mais tous les sujets éducatifs. En un mot, la mobilisation du rire par la médiation de la parrhêsia constitue une qualité de cet art, puisque le rire est propre à l'homme.

Si le rire est dangereux, il en va de même pour le sérieux qui inhibe l'affectivité, la détente et la jouissance de la vie intelectuelle, laquelle, tout en souriant, crie : sans rire, sans sagesse !

\section{REFERÊNCIAS}

ALBERTI, Verena. O riso e o risível: na história do pensamento. Rio de Janeiro: Jorge Zahar Ed., 2002.

ARAPIRACA, Mary de Andrade. Riso e educação: prólogo de uma paideia. Salvador: EDUFBA, 2017.

ARISTÓTELES. Partes dos animais. Lisboa: Imprensa Nacional-Casa da Moeda, 2010.

BERGSON Henri. Essai sur la signification du comique. 6 ed. Paris, PUF, 1991.

CICÉRON. Oeuvres complètes de Cicéron. Dialogues de l'Orateur. Paris: C.L.F. Panckoucke, 1830.

Disponível em: https://books.google.com.br/. Acesso em: 19 jul. 2019.

CITOT, Vincent. Entretien avec Michel Onfray. Le Philosophoire, n. 17, p. 9-14, 2002. Disponível em https://www.cairn.info/revue-le-philosophoire-2002-2-page-9.htm. Acesso em: 19 jul. 2019.

DESCARTES, René. Les passions de l'âme. Paris: Arvensa Éditions, 2015.

FOUCAULT, Michel. La parrêsia. Anabases. Traditions et réceptions de l'Antiquité, n. 16, p. I57- I88, 20 12. Disponível em: https://journals.openedition.org/anabases/3959. Acesso em: 19 jul. 2019.

FOUCAULT, Michel. Le gouvernement de soi et des autres. Cours au Collège de France. 1982-1983. Paris: EHESS, Gallimard, Seuil, 2008.

FOUCAULT, Michel. Fearless speech. Los Angeles:Semiotext(e), $200 \mathrm{I}$.

GLANSDORFF, Valérie. Michel Foucault: la "parrêsia" dans le gouvernement de soi et des autres. Revue de philosophie ancienne, v. 28, n. I, p. 67-84, 2010. Disponível em:

https://www.jstor.org/stable/24358467. Acesso em: 19 jul. 2019.

LARROSA Jorge. Pedagogia profana: danças, piruetas e mascaradas. 5 ed. Belo Horizonte: Autêntica, 2013.

MARQUES JUNIOR, Ivan Neves. O riso segundo Cícero e Quintiliano: tradução e comentários de De oratore, livro II, 2 16-29I (De ridiculis) e da Institutio Oratoria, Livro VI, 3 (De risu). Dissertação Universidade de São Paulo, São Paulo, 2008. Disponível em: https://teses.usp.br/teses/disponiveis/8/8 I43/tde- 13 102008- I54439/pt-br.php. Acesso em: 19 jul. 2019. 
MARTÍN, Xus. O senso de humor no trabalho com adolescentes com problemas de adaptação. In: ARANTES, Valéria Amorim (org.). Humor e alegria na educação. São Paulo: Summus, 2006. p. I 5 I 167.

MINOIS, Georges. História do riso e do escárnio. São Paulo: Unesp, 2003.

MONTAIGNE, Michel de. Essais. Paris: Gallimard, 2009.

PAGNI, Pedro Angelo. $O$ cuidado de si em Foucault e as suas possibilidades na educação: algumas considerações. In: SOUZA, Luís Antônio Francisco de; SABATINE, Thiago Teixeira; MAGALHÃES, Bóris Ribeiro de (orgs.). Michel Foucault: sexualidade, corpo e direito. Marília: Oficina Universitária; São Paulo: Cultura Acadêmica, 201 I. p. 19-45.

PAVIS, Patrice. Dicionário de teatro. São Paulo: Perspectiva, 1999.

PERELMAN, Chaïm; OLBRECHS-TYTECA, Lucie. Tratado da argumentação: a nova retórica. 2 ed. São Paulo: Martins Fontes, 2005.

POIZAT, Jean-Claude. Éloge du rire. Le Philosophoire, n 17, p. 3-8, 2002. Disponível em: https://www.cairn.info/revue-le-philosophoire-2002-2-page-3.htm?contenu=resume\#. Acesso em: 19 jul. 2019.

PROPP, Vladimir. Comicidade e riso. São Paulo. Ática, 1992.

QUINTILIEN. Quintilien et Pline le Jeune. Paris: J.J. Dubochet et Compagnie Éditeurs, I842. Disponível em:

https://books.google.com.br/books/about/Quintilien_et_Pline_Le Jeune.html?id=dadAAAAAcAAJ\&redir esc =y. Acesso em: 19 jul. 2019.

REBOUL, Olivier. Introdução à retórica. São Paulo: Martins Fontes, 2004.

SKINNER, Quentin. Hobbes e a teoria clássica do riso. São Leopoldo: Editora Unisinos, 2002.

VIEIRA, Antônio. Sermoens e Varios Discursos do P. Antonio Vieira. Lisboa: Valentim da Costa Deslandes, 17/0. Disponível em: https://digital.bbm.usp.br/handle/bbm/4580. Acesso em 19 jul. 2019. 